\title{
BOBINE SUPRACONDUCTRICE NON RAYONNANTE
}

\author{
P. GAYTE \\ Groupe d'Etudes Cryotechniques, \\ Centre d'Etudes et Recherches de la Compagnie Electro-Mécanique, \\ 55, av. Jean-Jaurès, 93-Le Bourget, France
}

\begin{abstract}
Résumé. - Le stockage de l'énergie dans une inductance supraconductrice non rayonnante présente de grands avantages. Parmi les différentes géométries possibles, les bobines sphériques ou ellipsoïdales avec écran électromagnétique sont très intéressantes. En effet, pour une même énergie stockée dans des conditions données (même induction, même contrainte sur le conducteur), les bobinés sphériques (ou ellipsoïdales) qui nécessitent approximativement la même quantité de supraconducteur, ont un encombrement hors tout très inférieur.

La réalisation d'une bobine supraconductrice ellipsoïdale, destinée à la mesure des pertes des grandes réactances shunt, a permis de vérifier expérimentalement les relations données pour ces types de bobinages.
\end{abstract}

Abstract. - Energy storage in a non radiating superconducting coil shows important advantages. Among the different possible geometries, spherical or ellipsoidal coils with electromagnetic screen are very interesting. In fact, for the same stored energy in the same conditions (field, stress on the conductor), spherical (or ellipsoidal) coils which need approximately the same amount of superconductor as other geometries, have the lowest external dimensions.

The making of a superconducting ellipsoidal coil designed for measuring the high power shunt reactance losses, has allowed the experimental verification of the described calculation method.

1. Introduction. - Le stockage d'énergie dans une inductance supraconductrice pourvue d'un écran électromagnétique constitue une solution très séduisante. L'absence de rayonnement électromagnétique extérieur permet de libérer rapidement l'énergie stockée sans perte dans les masses métalliques voisines. Les cryostats métalliques conventionnels sont alors utilisables.

La bobine réalisée, destinée à la mesure précise des angles de pertes de l'ordre de 2 à $3 \%$ des grandes réactances shunt et fonctionnant à 50 ou $60 \mathrm{~Hz}$, a servi en outre de modèle géométrique probatoire de bobine avec écran.

2. Les bobines non rayonnantes. Les bobines sphériques ou elliptiques: - 2.1 BOBINES NON RAYONNANTES. - Trois types de bobines non rayonnantes sont envisageables :

- Les bobines de forme classique (solénoïde) pourvues d'un écran électromagnétique.

- Les bobines toroïdales.

- Les bobines sphériques ou ellipsoïdales.

La recherche du coût minimum d'un dispositif de stockage d'énergie doit faire intervenir, entre autres, le prix de la bobine ainsi que le prix du cryostat et du système de réfrigération.

Pour une même énergie stockée $\left(10^{10}\right.$ et $\left.10^{13} \mathrm{~J}\right)$, une même valeur des forces de contraintes sur le conducteur et une même induction au centre, les quantités de supraconducteurs nécessaires sont voisines pour ces trois types de bobinages [1]

Mais l'encombrement hors tout est inférieur dans le cas des bobines sphériques ou elliptiques non rayonnantes [2].

2.2 BOBINES SPHÉRIQUES OU ELLIPTIQUES NON RAYONNANTES. - Le but est d'obtenir, à l'aide de deux enroulements coaxiaux de sens inverse, répartis sur deux sphères concentriques ou sur deux ellipsoïdes homofocaux, une induction nulle à l'extérieur du bobinage écran, tout en utilisant le supraconducteur le plus judicieusement possible (dimensionnement minimum, fonctionnement près des limites de ses possibilités) et en répartissant au mieux les efforts électrodynamiques.

Les deux enroulements sont parallèles aux plans équatoriaux des deux sphères concentriques de rayon $R_{1}$ (enroulement inducteur) et $R_{2}$ (enroulement écran) (Fig. 1).

Les densités de courant de surface sont :

$$
a_{1}=A_{1} \sin \theta
$$

pour la sphère intérieure,

$$
a_{2}=A_{2} \sin \theta
$$

pour la sphère extérieure.

L'induction à l'extérieur du système est nulle si on réalise la condition :

$$
A_{1} R_{1}^{3}=-A_{2} R_{2}^{3} .
$$




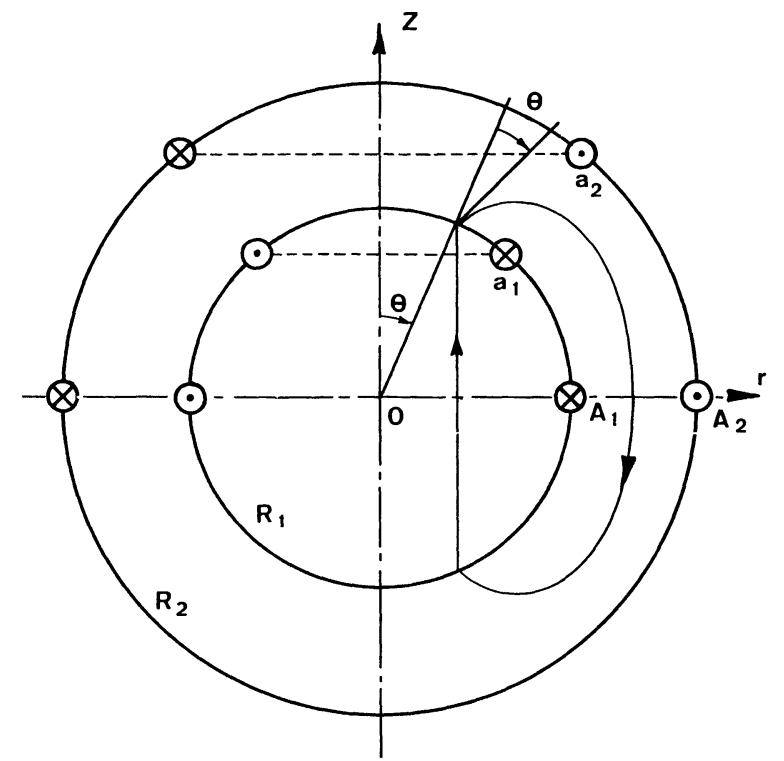

FIG. 1. - Bobine sphérique avec écran.

FIG. 1.1. - Schéma :

$$
\begin{aligned}
& a_{1}=A_{1} \sin \theta \\
& a_{2}=A_{2} \sin \theta \\
& A_{2}=-\frac{A_{1}}{4} .
\end{aligned}
$$

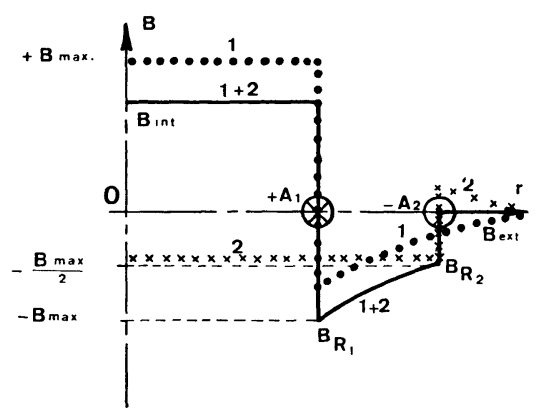

FIg. 1.2. - Tracé de l'induction le long d'un axe contenu dans le plan équatorial.

Le supraconducteur sera utilisé au mieux de ses performances si l'on s'impose la condition :

$$
B_{R_{1}}=B_{\text {int }}=B_{\max }
$$

où $B_{R_{1}}=$ induction sur la sphère de rayon $R_{1}$,

$B_{\text {int }}=$ induction dans la sphère intérieure,

$B_{\max }=$ induction maximale vue par le supraconducteur.

Cette condition est remplie si on choisit

$$
4 R_{1}^{3}=R_{2}^{3} \text {. }
$$

Le rayon $R_{2}$ est alors minimum pour un rayon $R_{1}$ donné.

En outre, dans le plan équatorial, l'induction de part et d'autre de la surface de la bobine interne ayant des valeurs égales en module, mais de sens opposé, l'équilibre des forces est alors réalisé.

Ces conditions ont été généralisées au cas des ellipsoïdes homofocaux. Les principaux résultats apparaissent dans le tableau I (les couches de courant ont été supposées infiniment minces dans le but de simplifier la formulation mathématique).
L'application de ces relations au cas réel de bobinages non rayonnants sphériques ou ellipsoïdaux d'épaisseur finie (constitués par exemple par des galettes de quelques centimètres d'épaisseur) (Fig. 2) a été effectuée au moyen de calculs sur ordinateur.

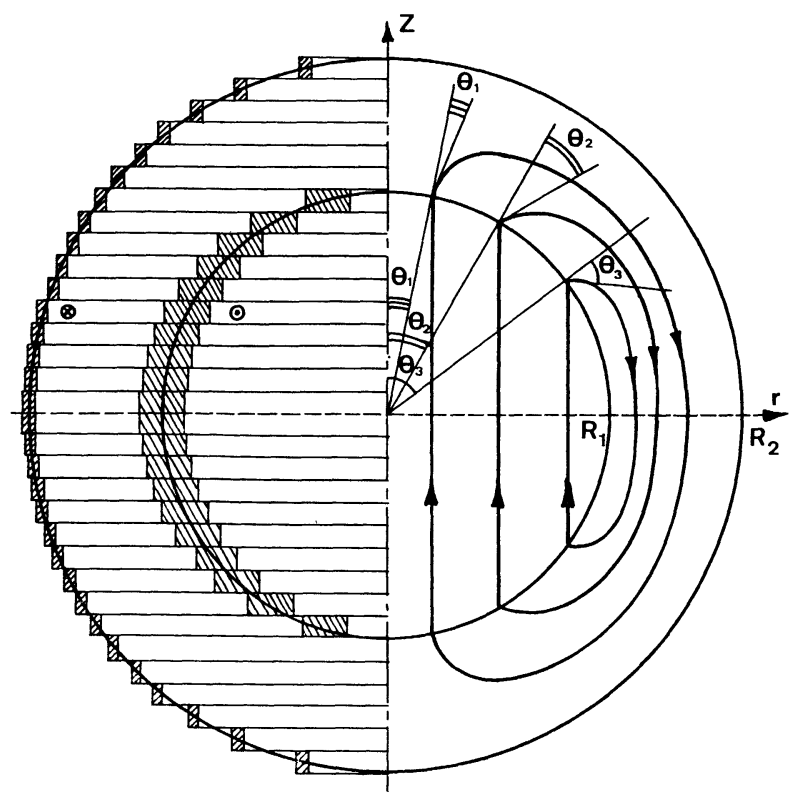

Fig. 2. - Exemple de réalisation d'une bobine sphérique non rayonnante.

Dans le cas où les différentes couches sont suffisamment divisées et correctement réparties sur les sphères ou les ellipsoïdes moyens, les calculs ont permis de vérifier que l'induction était négligeable à l'extérieur du système, même à quelques millimètres du bobinage écran.

2.3 EFFORTS ÉLECTRODYNAMIQUES DANS LES BOBINAGES SPHÉRIQUES [2], [3]. - Considérons une spire de la sphère intérieure coupant en $P$ le plan $Z O r$ (Fig. 3). L'induction moyenne au point $P$, résultant des inductions sur les surfaces intérieure et extérieure de la sphère, est dirigée suivant le rayon $O P$ vers l'extérieur.

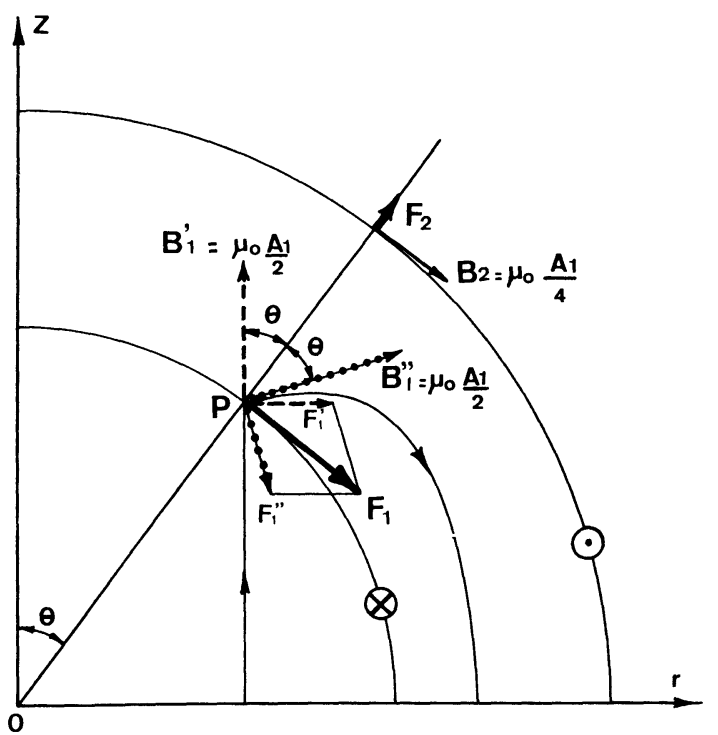

FIG. 3. - Répartition des forces sur la bobine intérieure et sur la bobine écran. 


\begin{tabular}{|c|c|c|}
\hline & $\begin{array}{l}\text { Bobines sphériques concentriques } \\
\text { (fig.1) }\end{array}$ & $\begin{array}{l}\text { Bobines ellipsoidales homofocales } \\
\text { (fig.12) }\end{array}$ \\
\hline $\begin{array}{l}\text { Paramètrcs } \\
\text { géométriques }\end{array}$ & $\begin{array}{l}R_{1}=\text { Rayon de la sphère intérieure } \\
(m) \\
R_{2}=\text { Rayon de la sphère extérieure } \\
(m)\end{array}$ & $\begin{array}{l}2 q_{1}: \text { Petit axe de l'ellipsoide intérieur } \\
(m) \\
2 q_{2}: \text { Petit axe de l'ellipsoide extérieur } \\
(m) \\
2 p_{1}: \text { Grand axe de l'ellipsoide intérieur } \\
(m) \\
2 p_{2}: \text { Grand axe de l'ellipsoide extérieur } \\
(m) \\
\mu_{1}: \text { Excentricité de l'ellipsoìde intérieur } \\
\mu_{2}: \text { Excentricité de l'ellipsoìde extérieur } \\
\mu=\frac{r_{1}+r_{2}}{2 c} \quad v=\frac{r_{1}-r_{2}}{2 c}\end{array}$ \\
\hline $\begin{array}{l}\text { Intensité du courant } \\
\text { dans les conducteurs } \\
\text { (A) }\end{array}$ & I & I \\
\hline Inductance $(\mathrm{H})$ & L & L \\
\hline $\begin{array}{l}\text { Induction dans le } \\
\text { volume intérieur } \\
\text { (Tesla) }\end{array}$ & $\begin{aligned} B_{\text {int }} & =\frac{2}{3} \mu_{0}\left(A_{1}+A_{2}\right) \\
& =\frac{1}{2} \mu_{0} A_{1} \\
\left(\mu_{0}\right. & \left.=4 \pi \cdot 10^{-7}\right)\end{aligned}$ & $\begin{array}{l}B_{\text {int }}=\frac{2}{3} \mu_{0}\left(A_{1}+A_{2}\right) \\
B_{\text {int }}=\frac{2 \mu_{0} A_{1}}{3}\left[1-\frac{\frac{2 \mu_{2}}{\mu_{2}^{2}-1}-\ln \frac{\mu_{2}+1}{\mu_{2}-1}}{\frac{2 \mu_{1}}{\mu_{1}^{2}-1}-\ln \frac{\mu_{1}+1}{\mu_{1}-1}}\right]\end{array}$ \\
\hline $\begin{array}{l}\text { Répartition } \\
\text { des nappes } \\
\text { superficielles } \\
\text { de courant } \\
\text { (A } / \mathrm{m})\end{array}$ & $\begin{array}{l}a_{1}=A_{1} \sin \theta \\
a_{2}=A_{2} \sin \theta\end{array}$ & $\begin{array}{l}a_{1}=\frac{A_{1}}{3} \frac{\sqrt{1-v^{2}}}{\sqrt{\mu_{1}^{2}-v^{2}}} \frac{1}{\frac{\mu_{1}}{2}-\frac{\mu_{1}^{2}-1}{4} \ln \frac{\mu_{1}+1}{\mu_{1}-1}} \\
a_{2}=\frac{A_{2}}{3} \frac{\sqrt{1-v^{2}}}{\sqrt{\mu_{1}^{2}-v^{2}}} \frac{1}{\frac{\mu_{2}}{2}-\frac{\mu_{2}^{2}-1}{4} \ln \frac{\mu_{2}+1}{\mu_{2}-1}}\end{array}$ \\
\hline $\begin{array}{l}\text { Conditions pour que } \\
\text { l'induction soit nulle } \\
\text { à l'extérieur du plus } \\
\text { grand volume }\end{array}$ & $A_{2}=-A_{1} \frac{R_{1}^{3}}{R_{2}^{3}}=$ & $A_{2}=-A_{1} \frac{\frac{2 \mu_{2}}{\mu_{2}^{2}-1}-\ln \frac{\mu_{2}+1}{\mu_{2}-1}}{\frac{2 \mu_{1}}{\mu_{1}^{2}-1}-\ln \frac{\mu_{1}+1}{\mu_{1}-1}}$ \\
\hline $\begin{array}{l}\text { Énergie stockée } \\
\text { (Joules) }\end{array}$ & $W m=\frac{4 \pi}{9} \mu_{0} A_{1}^{2} R_{1}^{3}$ & $W m=\frac{16}{27} \mu_{0} \pi C^{3} \frac{A_{1}\left(A_{1}+A_{2}\right)}{\frac{2 \mu_{1}}{\mu_{1}^{2}-1}-\ln \frac{\mu_{0}+1}{\mu_{1}-1}}$ \\
\hline $\begin{array}{l}\text { Dimensions } \\
(\mathrm{m})\end{array}$ & $R_{1}=\left(\frac{3 L I^{2}}{2 \pi \mu_{0} A_{1}^{2}}\right)^{1 / 3}$ & $\begin{array}{l}C=\left(\frac{3}{2} \frac{L I^{2} \mu_{0}}{\left.4 \pi B_{\text {int }}^{2} \frac{\mu_{1}\left(\mu_{1}^{2}-1\right)}{\frac{P_{2}=\mu_{2} C}{P_{1}}}\right)^{1 / 3}}\right. \\
P_{1}=\mu_{1} C\end{array}$ \\
\hline & $R_{2}=1,587 R_{1}$ & $q_{1}=c \sqrt{\mu_{1}^{2}-1} \quad q_{2}=c \sqrt{\mu_{2}^{2}-1}$ \\
\hline $\begin{array}{l}\text { Longueur des } \\
\text { conducteurs } \\
\text { sur chacune } \\
\text { des bobines } \\
\text { (m) }\end{array}$ & $\begin{array}{l}I_{1}=\frac{\pi^{2} A_{1} R_{1}^{2}}{I} \\
I_{2}=\frac{\pi^{2} A_{2} R_{2}^{2}}{I}\end{array}$ & $\begin{array}{l}I_{1}=\frac{\pi^{2}}{I} \frac{A_{1}}{3} C^{2} \frac{V \mu_{1}^{2}-1}{\frac{\mu_{1}}{2}-\frac{\mu_{1}^{2}-1}{4} \ln \frac{\mu_{1}+1}{\mu_{1}-1}} \\
I_{2}=\frac{\pi^{2}}{I} \frac{A_{2}}{3} C^{2} \frac{\sqrt{\mu_{2}^{2}-1}}{\frac{\mu_{2}}{2}-\frac{\mu_{2}^{2}-1}{4} \ln \frac{\mu_{2}+1}{\mu_{2}-1}}\end{array}$ \\
\hline
\end{tabular}

TABLEAU I

Principales formules caractéristiques des bobines sphériques et ellipsoïdales non rayonnantes 
La force élémentaire $F_{1}$ sur la spire s'exerce alors tangentiellement à la sphère inductrice. La bobine intérieure est donc soumise à des efforts d'éclatement parallèles au plan équatorial et à des efforts de compression perpendiculaires à ce dernier.

La force élémentaire $F_{1}$ a pour expression :

$$
F_{1}=\frac{\mu_{0}}{2} R_{1} A_{1}^{2} \sin 2 \theta \mathrm{d} l \mathrm{~d} \theta
$$

avec $\mu_{0}=4 \pi \cdot 10^{-7}$.

$A_{1}=$ densité de courant par unité de longueur d'arc méridien $(\mathrm{A} / \mathrm{m})$;

$\theta=$ angle polaire ;

$\mathrm{d} \theta=$ élément d'angle polaire correspondant à la spire ;

$\mathrm{d} l=$ longueur élémentaire de la spire.

Cette force est nulle aux pôles et à l'équateur. Elle est maximale pour $\theta=45^{\circ}$.

Sur le bobinage écran, l'induction étant tangentielle, la force $F_{2}$ est dirigée vers l'extérieur suivant le rayon. Le bobinage écran est donc soumis à un effort d'éclatement parallèle au plan équatorial, et à un effort tendant à séparer les deux hémisphères. La force élémentaire $F_{2}$ a pour expression :

$$
F_{2}=\frac{\mu_{0}}{16} R_{1} A_{1}^{2} \sin \theta \mathrm{d} \theta \mathrm{d} l \text {. }
$$

Elle est nulle aux pôles et maximale à l'équateur.

Son amplitude est 8 fois plus faible que sur la bobine intérieure.

De telles géométries de bobinage [4] permettent d'envisager le stockage de l'énergie avec une densité voisine de $10 \mathrm{MJ} / \mathrm{m}^{3}$ [3].

3. Buts de la bobine supraconductrice ellipsoïdale réalisée. - 3.1 MESURE PRÉCISE DE L'ANGLE DE PERTES DES GRANDES RÉACTANCES SHUNT. - Les grandes réactances shunt, utilisées pour la compensation de l'effet capacitif par rapport à la terre des longues lignes à très haute tension, ont des pertes actives correspondant à 2 ou $3 \%$ de leur puissance réactive.

La mesure au pont de ces pertes est peu précise du fait de l'erreur d'angle commise en utilisant des transformateurs de courant.

La détermination précise se fait par voie calorimétrique. Mais cette méthode étant longue et coûteuse, l'étalonnage porte sur l'un des appareils d'une même série qui sert alors de référence à la mesure au pont des autres réactances.

En perfectionnant la technique de mesure au pont, les longues durées d'échauffement peuvent être évitées [5]. La réactance à mesurer constitue tout d'abord l'une des branches d'un pont dont on réalise l'équilibre (Fig. 4). Puis on la remplace par une bobine supraconductrice dont les caractéristiques sont presque idéales. Les seules pertes manquantes pour rétablir l'équilibre du pont peuvent alors être engendrées grâce à une résistance ajustable avec précision connectée en parallèle sur la bobine supraconductrice (Fig. 5).

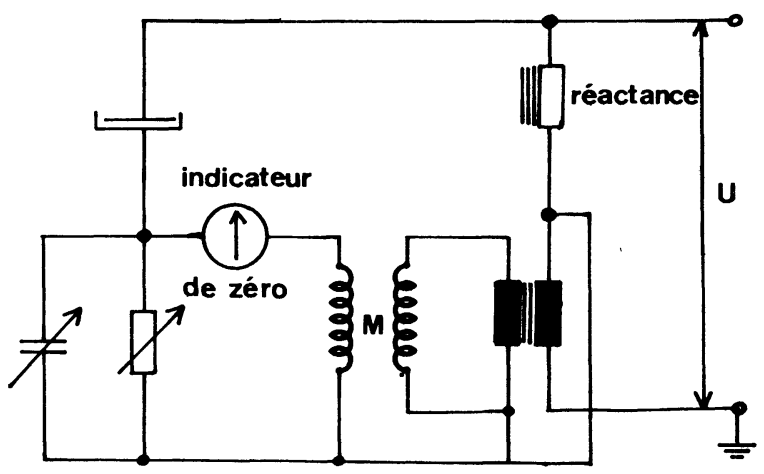

(a)

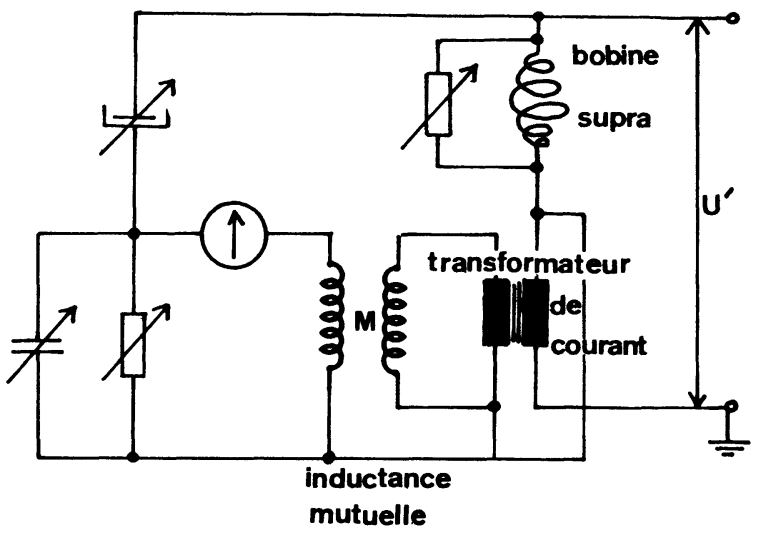

(b)

Fig. 4. - Schéma de l'installation de mesures des pertes.

3.2 Modèle GÉOMÉTRIQUe PROBATOIRE. - Ce modèle permet de vérifier expérimentalement la validité de la méthode de calcul des enroulements avec écran de ce type.

4. Réalisation de la bobine supraconductrice ellipsoïdale [6] (Fig. 6) (photo 1). - Avec le même rapport "puissance active/puissance réactive » $(\operatorname{tg} \delta)$ et même courant $(100,200$ et $400 \mathrm{~A})$ que les grandes réactances shunt, mais avec des tensions et puissances fortement réduites, une bobine supraconductrice peut présenter des pertes négligeables $\left(\operatorname{tg} \delta_{L} \simeq 10^{-5}\right)$ si elle est correctement dimensionnée. Il faut cependant tenir compte des pertes ohmiques dans les amenées de courant à la bobine qui doivent être suffisamment faibles.

4.1 CHOIX DU MATÉRIAU SUPRACONDUCTEUR. Des mesures de pertes en régime alternatif ont été effectuées sur un grand nombre d'échantillons de nature et d'origines diverses par la méthode calorimétrique ( $\mathrm{Nb}$ - $\mathrm{Ti}$ monobrin, $\mathrm{Nb}$-Ti multifilamentaire avec matrice de cuivre ou de cupro-nickel, $\mathrm{Nb}_{3} \mathrm{Sn}$ en ruban, $\mathrm{Nb}$ monobrin...).

A performances égales (courant critique supérieur ou égal à $100 A_{\text {eff }}$ pour une induction extérieure de $1000 \mathrm{G}$ crête), le niobium présente les pertes minimales.

Le supraconducteur retenu est un fil de niobium de $0,8 \mathrm{~mm}$ de diamètre, de bonne pureté $(99,9 \%)$, poli 


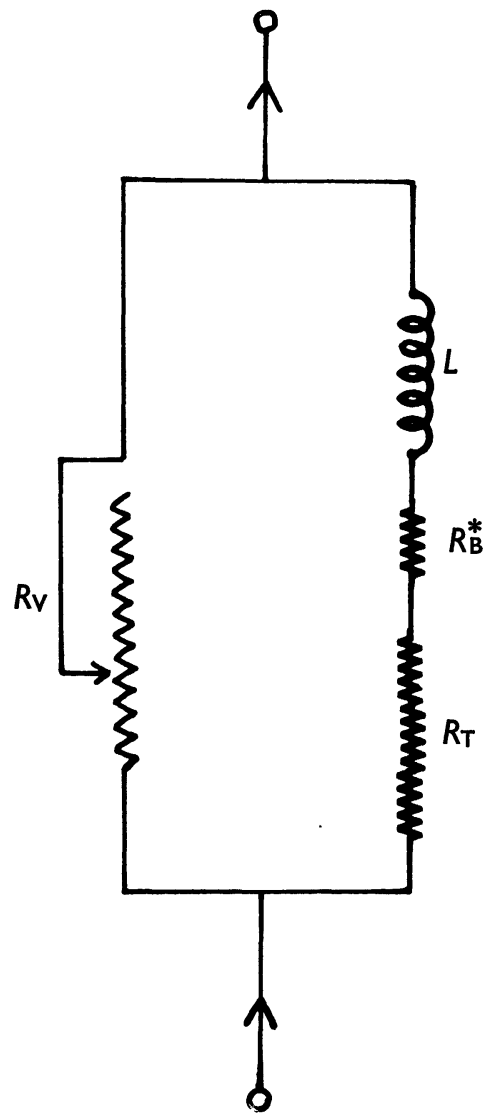

FIG. 5. - Schéma équivalent du dispositif d'étalonnage de l'installation de mesure des portes des réactances shunt :

$$
\begin{gathered}
\operatorname{tg} \delta \approx \frac{R_{\mathrm{T}}}{L \omega}+\frac{L \omega}{R_{\mathrm{V}}} \\
\operatorname{tg} \delta_{\mathrm{L}}=\frac{R_{\mathrm{B}}}{L \omega} \\
R_{\mathrm{T}} \ll R_{\mathrm{V}} \\
R_{\mathrm{B}} \\
\operatorname{tg} \delta_{\mathrm{L}}
\end{gathered}
$$

$R_{\mathrm{T}}$ Résistance des amenées de courant.

$R_{\mathrm{B}}^{*}$ « Résistance » apparente de la bobine supraconductrice.

$L$ Inductance de la bobine supraconductrice.

$R_{\mathrm{V}}$ Résistance variable.

$\delta_{\mathrm{L}}$ Angle de pertes de la bobine.

$\delta$ Angle de pertes correspondant au schéma équivalent.

et recuit $2 \mathrm{~h}$ sous vide à $950^{\circ} \mathrm{C}$. Les caractéristiques critiques de ce fil apparaissent figure 7 et les pertes à $50 \mathrm{~Hz}$ sont données figure 8 .

4.2 Forme ET DIMENSIONS DE LA BOBINE. - Deux paramètres sont imposés; le courant dans la bobine $\left(I_{\text {eff }}=100 \mathrm{~A}\right)$ et l'inductance $(L \simeq 7 \mathrm{mH})$. Compte tenu de l'angle de pertes imposé pour la bobine $\left(\operatorname{tg} \delta \simeq 10^{-5}\right.$ ) et des pertes de l'échantillon mesurées à $50 \mathrm{~Hz}$, l'induction au centre ne doit pas dépasser $800 \mathrm{G}$ environ (cette limite dépend naturellement de la géométrie des bobinages considérés).

Un calcul d'optimisation tenant compte des pertes en régime variable du fil supraconducteur et de son prix, du volume d'hélium nécessaire à chaque essai et du coût de la partie cryogénique montre que la forme

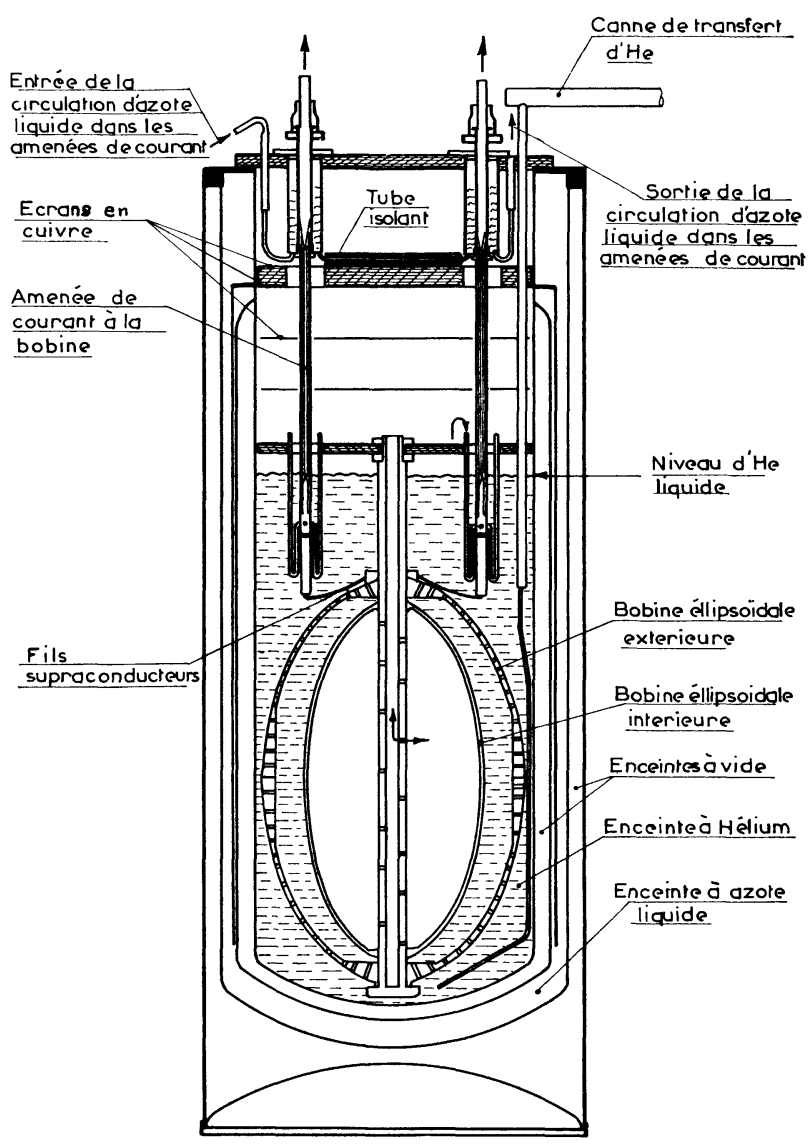

Fig. 6. - Vue d'ensemble de la bobine et de son cryostat.

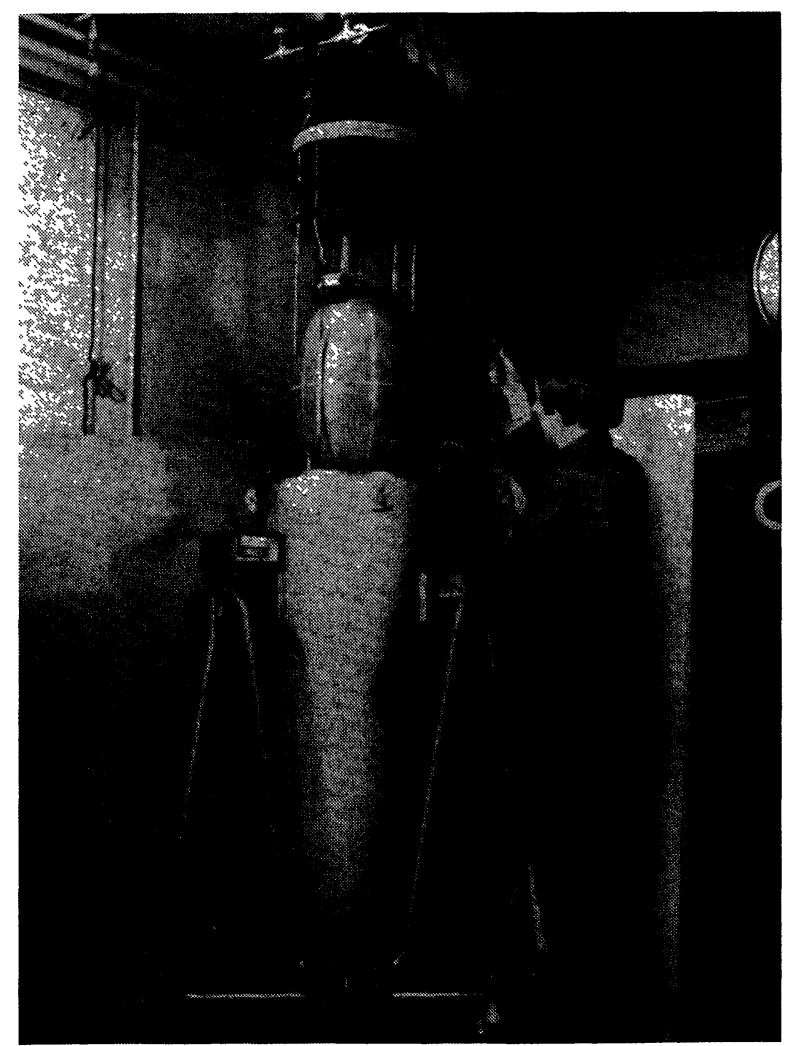

Рното 1. - Bobine supraconductrice ellipsoïdale. 


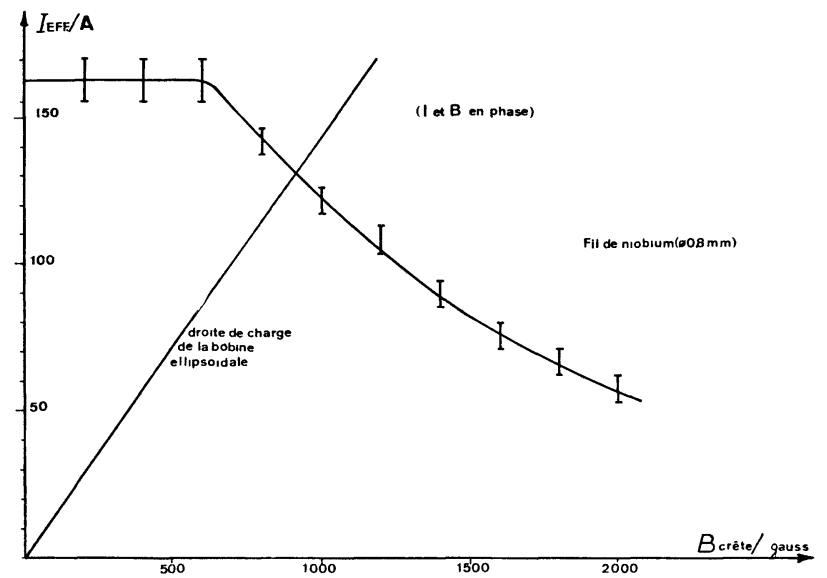

Fig. 7. - Caractéristiques critiques en échantillon court du fil de niobium utilisé.

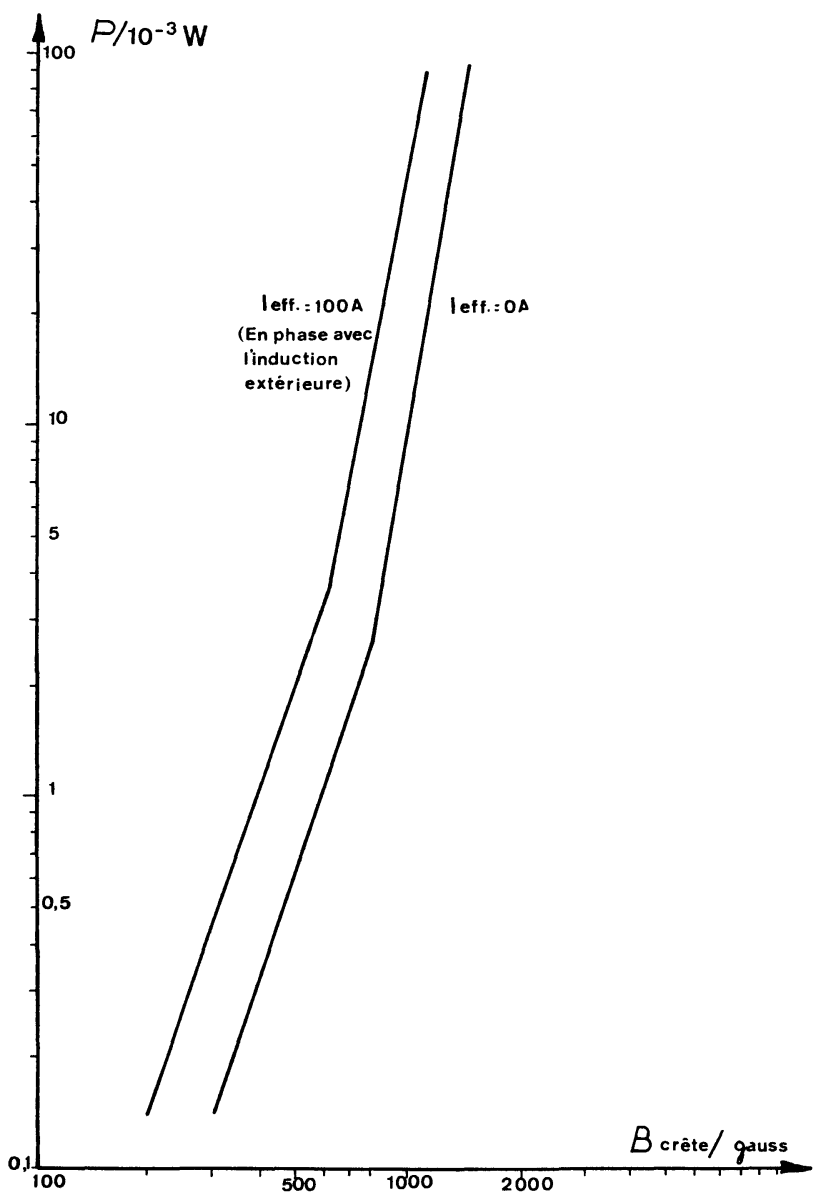

Fig. 8. - Pertes à $50 \mathrm{~Hz}$ du fil de niobium $(\varnothing=0,8 \mathrm{~mm})$ placé dans une induction extérieure (en phase avec le courant parcourant le fil supraconducteur).

ellipsoïdale est préférable aux géométries sphériques ou toroïdales qui sont beaucoup plus encombrantes.

Afin d'obtenir le meilleur échange thermique possible avec le bain, le conducteur n'est pas isolé ; il est bobiné en une seule couche.

Les dimensions principales du modèle géométrique réalisé apparaissent sur la figure 9.

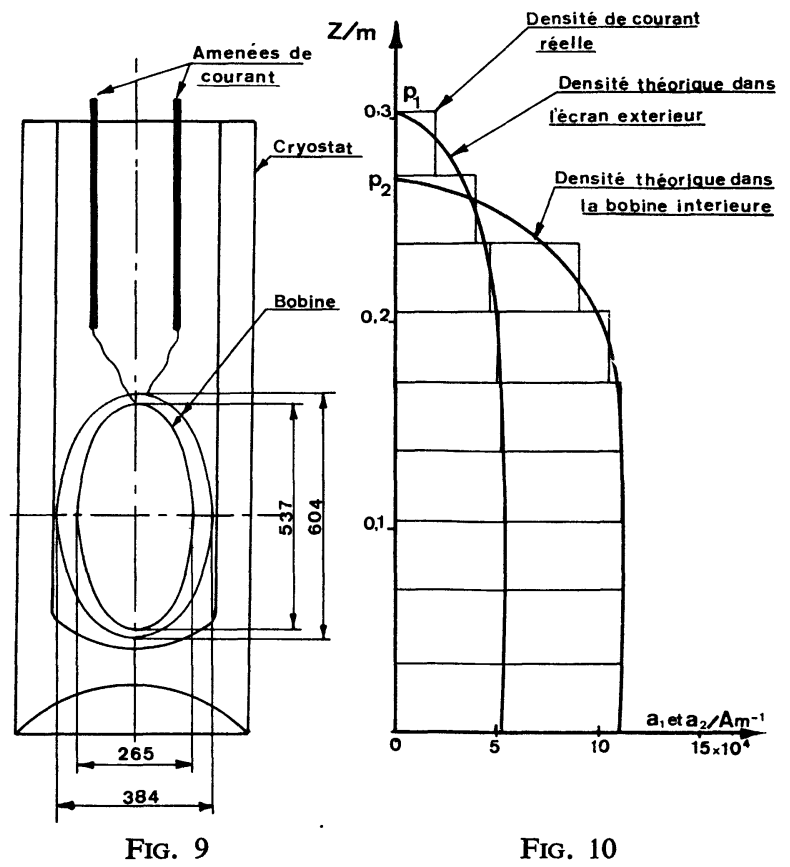

FIG. 9. - Schéma de la bobine ellipsoïdale expérimentale.

FIG. 10. - Densité de courant dans chacun des enroulements de la bobine ellipsoïdale.

Elles correspondent à un rapport longueur/diamètre de 1,57 et à un supplément de longueur de supraconducteur de $31 \%$ par rapport à la bobine sphérique. En contrepartie le cryostat a un volume $45 \%$ plus faible.

La densité de courant sur les deux ellipsoïdes est représentée figure 10 en fonction de la position axiale. En fait, la répartition réelle est discontinue pour des questions de fabrication.

4.3 DESCRIPTION DE LA BOBINE. - Le mandrin de la bobine est constitué par deux ellipsoïdes homofocaux réalisés en verre époxy (photo 2).

La teneur en verre est supérieure à $80 \%$ pour éviter toute détérioration lors de la mise en froid. La dilatation linéaire moyenne d'un tel matériau est alors

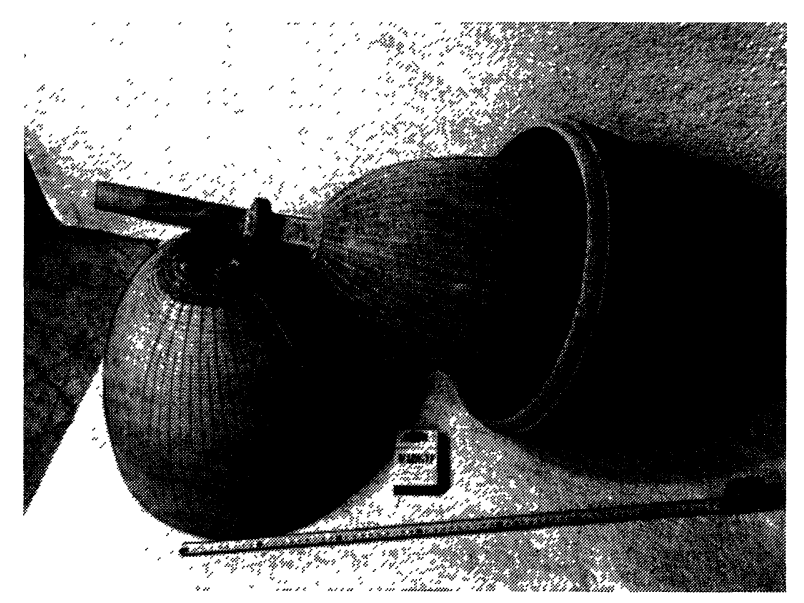

Рното 2. - Mandrins de la bobine réalisés en verre époxy. 
particulièrement intéressante ; elle est comparable à celle de l'acier 304 (acier au nickel-chrome).

L'ellipsoïde extérieur est en deux parties démontables qui se positionnent en $1 / 10^{\mathrm{e}}$ de millimètre par rapport à l'ellipsoïde intérieur lui-même collé sur un tube de verre époxy. La surface de chacun d'eux comporte des gorges circulaires parallèles destinées à recevoir les fils supraconducteurs. Perpendiculairement à ces dernières, des canaux sont aménagés pour permettre le refroidissement. Dans toute son épaisseur, l'ellipsoïde externe est percé de nombreux trous permettant à l'hélium liquide de pénétrer dans le volume intermédiaire et à l'hélium gazeux de s'en échapper.

L'enroulement est constitué par 4 fils de niobium bobinés en parallèle sur le mandrin. Chaque fil décrit un quart de circonférence puis passe à la gorge suivante en empruntant successivement chacune des 4 rainures méridiennes (photo 3) pour décrire à nouveau un quart de circonférence, etc...

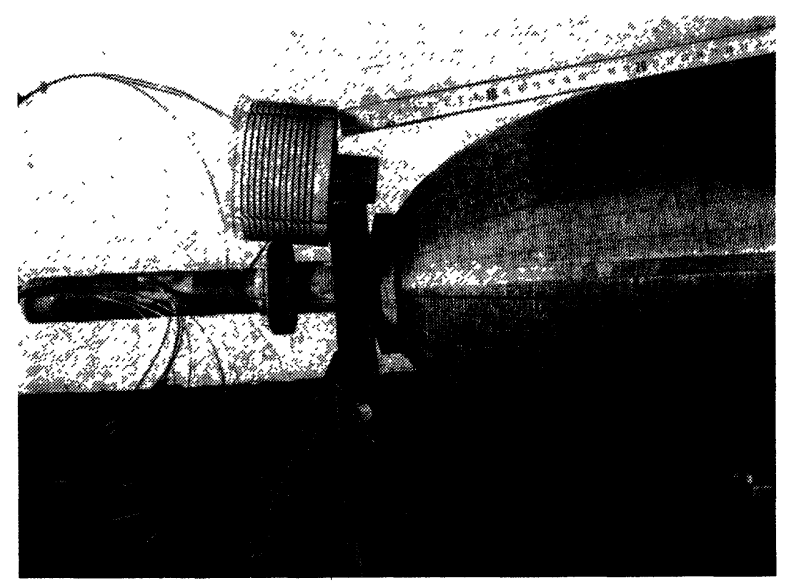

Pното 3. - Détails du bobinage.

Les fils sont maintenus dans leurs gorges par des frettes constituées par des rubans de mylar. Les 4 fils peuvent être associés en série, en parallèle ou en série parallèle.

4.4 Performances DE LA Bobine. - Mesure de l'induction. - La configuration de cette bobine ne permet pas la mesure de l'induction en tous les points $\mathrm{du}$ volume intérieur.

La mesure de l'induction a été effectuée sur l'axe de la bobine ainsi qu'à l'extérieur de l'ensemble grâce à une petite bobine de cuivre.

Les résultats expérimentaux corroborent à la sensibilité des mesures près (supérieure à $5 \%$ ), les résultats prévus par le calcul.

- Mesure des pertes à $50 \mathrm{~Hz}$. - Les pertes de la bobine ont été mesurées par la méthode calorimétrique et sont représentées (Fig. 11).

- Transition de la bobine. - La transition de la bobine se produit pour un courant de $130 A_{\text {eff }}$. Or, la droite de charge (Fig. 7) coupe la courbe de caractéristiques critiques $(I, B)$ en échantillon court à une valeur très voisine. Ceci vérifie donc expérimentalement

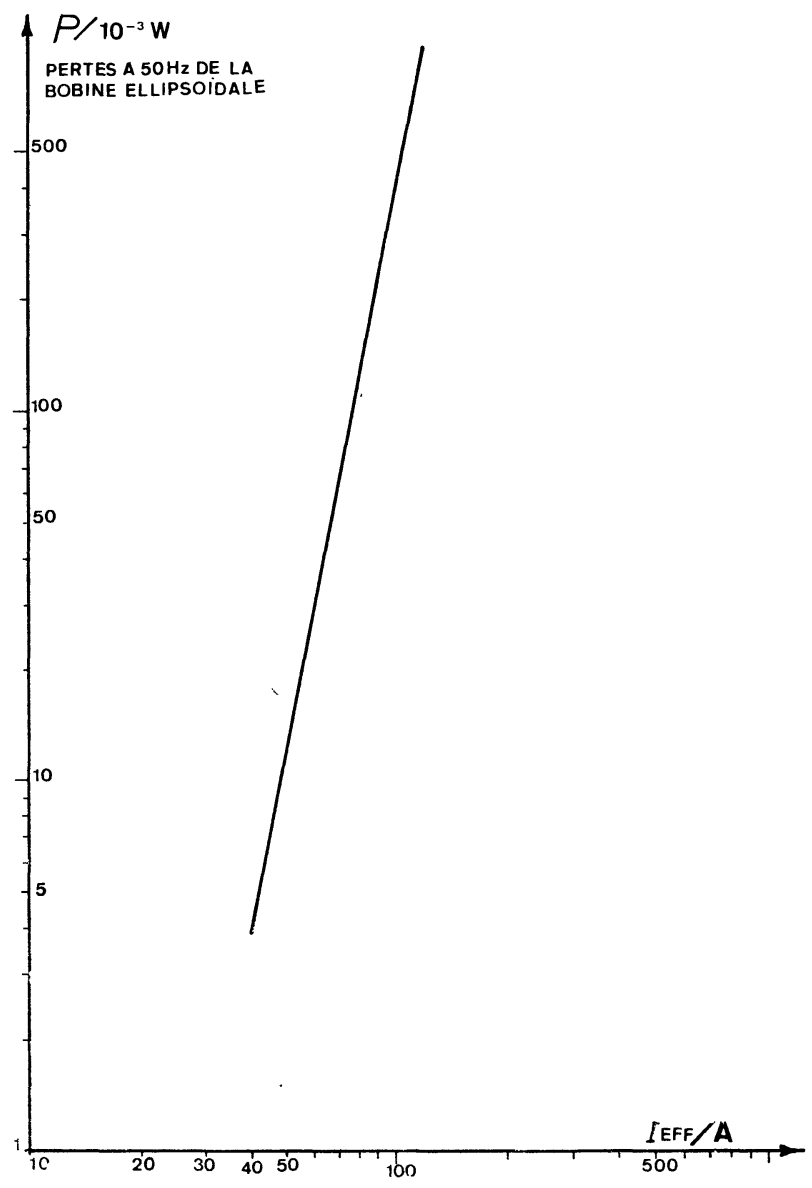

Fig. 11. - Pertes à $50 \mathrm{~Hz}$ de la bobine ellipsoïdale en fonction du courant.

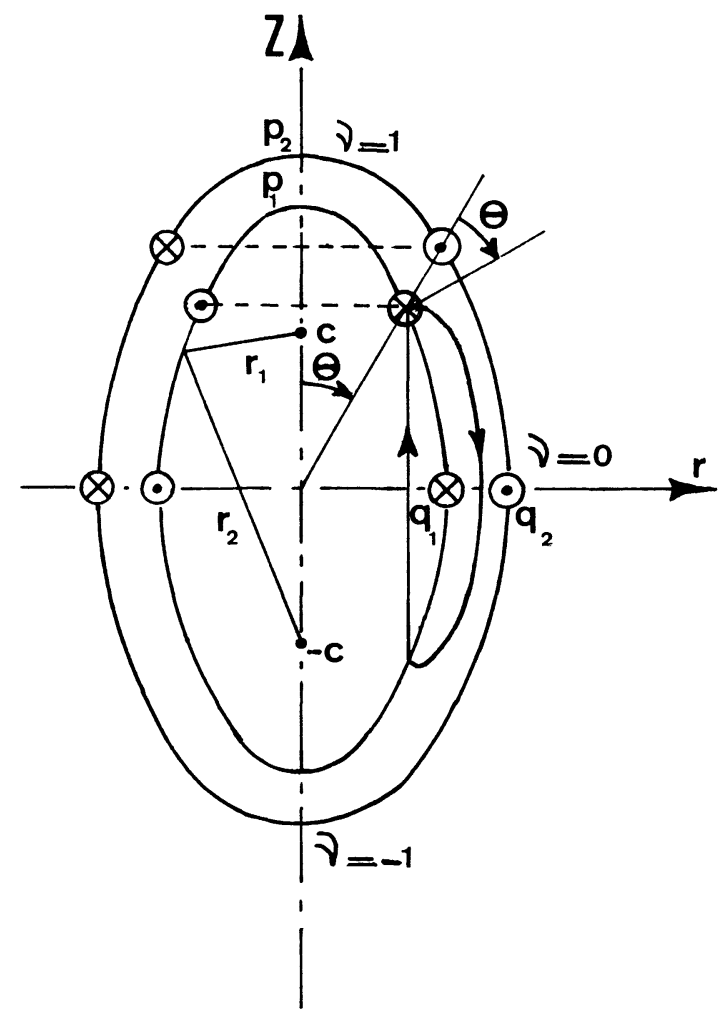

Fig. 12. - Bobine ellipsoïdale avec écran. 
le fait que, dans une telle géométrie, le supraconducteur est très bien utilisé car il travaille très près des limites de ses possibilités.

4.5 Protection De LA Bobine. - Un dispositif permet de détecter l'apparition d'une zone résistive dans la bobine.

5. Réalisation des amenées de courant. - Le problème est différent de celui des amenées de courant classiques pour lesquelles on cherche avant tout à limiter l'apport de chaleur en bain.

Dans le cas présent, il s'agit essentiellement d'obtenir une résistance électrique faible compatible avec l'utilisation envisagée, tout en limitant au mieux l'apport de chaleur au bain.
Une solution a pu être trouvée en refroidissant le haut des amenées de courant par une circulation d'azote liquide. Le corps de l'amenée, constitué par une multitude de petits fils de cuivre argentés, est lui-même refroidi par l'hélium vaporisé dans le bain. La résistance des deux amenées est maintenue pratiquement constante $(50 \mu \Omega)$, quel que soit le niveau d'hélium liquide, par adjonction, dans le bas des amenées, de tubes de cuivre. La partie inférieure coudée de ceux-ci plonge dans le bain (même lorsque le niveau d'hélium est au minimum). L'hélium vaporisé circule dans ces tubes, puis parvient au bas du corps des amenées de courant. L'apport de chaleur au bain par ces deux amenées est alors de $1,7 \mathrm{~mW} / \mathrm{A}$.

\section{Bibliographie}

[1] Brechna, H., Arendt, F. et Heinz, W., " Superconducting magnetic energy storage systems $»$. The Fourth International Conference on Magnet Technology, September 19-22, 1972.

[2] Sole, J. et Malandain, A., Techniques CEM nº 84 (mai 1972) 9-15.

[3] Gayte, P., Girard, B. et Malandain, A., Communication présentée à NATO, Advisory Group For Aerospace Research and Development, US, Air Force
Academy, Colorado Springs, Colorado, USA, 1215 juin 1972.

[4] Favereau, J., Brevet Français no 1516 849. «Dispositif de production de champ magnétique intense et uniforme dans un volume ellipsoïdal de révolution. "

[5] Sarbach, E., Tan, T. T., Brevet Suisse no 528087 : " Verfahren zur Messung der Wirkverluste Grosser Drosselspule sowie Mittel zu dessen Ausführung. "

[6] GAYTE, P., Techniques CEM nº 87, juin 1973. 